\title{
Three-dimensional changes in head shape after extended sagittal strip craniectomy with wedge ostectomies and helmet therapy
}

\author{
Pang-Yun Chou, MD,, ${ }^{1,2}$ Rami R. Hallac, PhD, ${ }^{1,3}$ Shitel Patel, MD, ${ }^{1}$ Min-Jeong Cho, MD, ${ }^{1}$ \\ Neil Stewart, MS, ${ }^{1}$ James M. Smartt, MD, ${ }^{1}$ James R. Seaward, MD, ${ }^{1}$ Alex A. Kane, MD,, ${ }^{1,3}$ and \\ Christopher A. Derderian, MD1
} 1Department of Plastic Surgery, UT Southwestern; ${ }^{3}$ Analytical Imaging and Modeling Center, Children's Medical Center, Dallas,
Texas; and ${ }^{2}$ Department of Plastic and Reconstructive Surgery, Chang Gung Memorial Hospital, Taoyuan, Taiwan

\begin{abstract}
OBJECTIVE Outcome studies for sagittal strip craniectomy have largely relied on the 2D measure of the cephalic in$\operatorname{dex}(\mathrm{Cl})$ as the primary indicator of head shape. The goal of this study was to measure the 2D and 3D changes in head shape that occur after sagittal strip craniectomy and postoperative helmet therapy.

METHODS The authors performed a retrospective review of patients treated with sagittal strip craniectomy at their institution between January 2012 and October 2015. Inclusion criteria were as follows: 1) isolated sagittal synostosis; 2) age at surgery < 200 days; and 3) helmet management by a single orthotist. The $\mathrm{Cl}$ was calculated from 3D images. Color maps and dot maps were generated from 3D images to demonstrate the regional differences in the magnitude of change in head shape over time.
\end{abstract}

RESULTS Twenty-one patients met the study inclusion criteria. The mean $\mathrm{Cl}$ was 71.9 (range 63.0-77.9) preoperatively and 81.1 (range 73.0-89.8) at the end of treatment. The mean time to stabilization of the $\mathrm{Cl}$ after surgery was $57.2 \pm 32.7$ days. The mean maximum distances between the surfaces of the preoperative and 1-week postoperative and between the surfaces of the preoperative and end-of-treatment 3D images were $13.0 \pm 4.1 \mathrm{~mm}$ and $24.71 \pm 6.83 \mathrm{~mm}$, respectively. The zone of maximum change was distributed equally in the transverse and vertical dimensions of the posterior vault.

CONCLUSIONS The $\mathrm{CI}$ normalizes rapidly after sagittal strip craniectomy ( 57.2 days), with equal distribution of the change in $\mathrm{Cl}$ occurring before and during helmet therapy. Three-dimensional analysis revealed significant vertical and transverse expansion of the posterior cranial vault. Further studies are needed to assess the 3D changes that occur after other sagittal strip craniectomy techniques.

https://thejns.org/doi/abs/10.3171/2017.1.PEDS16660

KEY WORDS sagittal craniosynostosis; surgical outcome; molding helmet; wedge ostectomies; 3D imaging; strip craniectomy; craniofacial

$\mathrm{S}$ aGitTal strip craniectomy is an established and effective treatment for sagittal synostosis. Unlike early strip craniectomy procedures, ${ }^{8,9}$ the techniques employed today utilize a variety of modifications to augment the changes in head shape achieved after surgery. These modifications include minimally invasive approaches, extending the width of the strip craniectomy, adding wedge ostectomies or barrel staves, and/or inserting cranial springs to facilitate lateral movement of the parietal bones. ${ }^{1,4-6,12-15,17}$ The most common postoperative adjunct is the wearing of a helmet to harness the rapid growth of the brain to augment the "passive" changes achieved in the shape of the cranial vault. $3,5,6,10$

Some groups have reported 3D analysis of cranial morphology and changes after open cranial vault treatment of sagittal synostosis; ${ }^{7,11,16}$ however, the outcome of sagittal strip procedures has primarily been reported utilizing the cephalic index (CI) ${ }^{1,3,6,10,13,15}$ We retrospectively reviewed the outcomes of extended sagittal strip craniectomy together with wedge ostectomies and postoperative helmet 
therapy by using 3D analysis to assess the distribution and speed of the change in head shape that occurs after surgery.

\section{Methods}

The UT Southwestern Medical Center and Children's Medical Center institutional review boards approved this retrospective study of all patients who had been treated with extended sagittal strip craniectomy at the Children's Medical Center in Dallas, Texas, between January 2012 and October 2015. Inclusion criteria were as follows: a diagnosis of nonsyndromic, isolated sagittal synostosis; treatment with sagittal strip craniectomy in patients younger than 200 days, age-corrected for preterm delivery; postoperative helmet management by a single orthotist; and completion of the prescribed course of helmet therapy until the age of at least 12 months. Collected demographic data included patient age at surgery, race, and sex. The surgical procedure for extended strip craniectomy with wedge ostectomies was modified from the original description by Jimenez et al. ${ }^{6}$ The technique is performed using two 3- to 4-cm incisions placed at the anterior and posterior limits of the synostotic sagittal suture. As described by others, ${ }^{15,17}$ we find that an endoscope is not necessary and we perform our procedure under direct visualization using lighted retractors and a head light. A 5-cm-wide strip craniectomy is centered on and incorporates the closed sagittal suture. One-centimeter-wide wedge ostectomies are performed under direct visualization just posterior to the coronal sutures and anterior to the lambdoid sutures, all directed toward the squamosal suture. The anterior wedge ostectomies extend up to but not beyond the temporalis muscle. The posterior wedge ostectomies extend up to or close to the squamosal suture.

Three-dimensional images were captured throughout treatment by using a laser scanner (STARscanner, Orthomerica). The mean time before surgery for the preoperative scan was 23 days (range 1-71 days). The 1-week postoperative scan was captured for helmet production. The end-of-treatment scan was captured at the last orthotist visit. Patient age at the end of helmet therapy ranged between 12 and 18 months depending on the provider.

The CI was calculated from measurements obtained from 3D images captured at all time points. A paired t-test was performed to compare the preoperative and end-oftreatment cranial indices. A $p$ value $<0.05$ was significant. MATLAB (MathWorks Inc.) was used to curve fit exponential regressions from time point measurements to identify the time point of head shape stability. This time point was identified when the curve hit $99.99 \%$ of the maximum change. The mean of all patients' time points to a stable CI was used as the time of head shape stability. End-of-treatment results with regard to $\mathrm{CI}$ were classified as excellent $(\mathrm{CI}>80)$, good $(\mathrm{CI} 75-80)$, or poor $(\mathrm{CI}<75)$.

Head scans at 3 different time points were used for 3D analysis: preoperatively, prehelmet (1 week postsurgery), and 1 year postoperatively (end of treatment). To generate a composite image from all subjects at each time point, a 3D template with known polygonal mesh connectivity was created from a 3D scan from a healthy subject.
For each 3D image, registration was performed through rigid translation and rotation to match the template using 25 landmarks placed at recognizable anatomical locations. In addition, anisotropic scaling of the template was performed to match each individual 3D image. Then, the template was deformed to each 3D scan using a thin-plate spline algorithm and closest point deformation. In this way, the polygonal indexing of all the 3D images matches that of the template, allowing for the creation of a mean (composite) head from the deformed templates by pointwise averaging. Thus, a composite head was created by combining the $3 \mathrm{D}$ deformed templates of all patients at each time point.

Color maps were generated to show the distance between the surfaces of the composite 3D images to show the $3 \mathrm{D}$ changes of the head and their magnitude over time. Color maps were designed to show increased distance in warm colors and decreased distance in cool colors. Analysis was performed using proprietary programs written in MATLAB.

To determine the location of maximal change for each subject at the prehelmet and end-of-treatment time points, a dot map in blue and red was created on the composite image.

\section{Results}

Twenty-one patients met the study inclusion criteria. Most of the patients were male (71\%). Fifty-seven percent of the patients were white, $38 \%$ were Hispanic, and 5\% were African American. The mean age at surgery was $113.6 \pm 27.9$ days (mean \pm standard deviation, range 83-202 days). The mean CI before surgery was $71.9 \pm 3.8$ (range 63.0-77.9) and at the end of treatment was $81.1 \pm$ 4.1 (range 73.0-89.8; Fig. 1). The average duration of postoperative helmet therapy was $327.5 \pm 27.9$ days (range 132-514 days).

To determine the change in CI from the surgical procedure alone (prehelmet) and the change in CI during helmet therapy, we measured the CI on 3D images obtained preoperatively, 1 week postoperatively (prehelmet), and at subsequent visits during helmet therapy. Figure 1 shows that the mean change in the $\mathrm{CI}$ at the end of treatment was $9.2 \%$ (range $1.5 \%-18.3 \%, \mathrm{p}<0.05$ ). The mean change in the $\mathrm{CI}$ from the preoperative image to the 1-week postoperative image (prehelmet scan) was 4.6. The mean change in the CI during helmet therapy was also 4.6. The average time from surgery to stabilization of the CI was $57.2 \pm$ 32.7 days (Fig. 2). Representative before and after photographs of a patient with a starting CI of 71 and an end-oftreatment CI of 80 are featured in Fig. 3. Treatment outcomes were categorized based on the CI for each patient at the end of treatment. Sixty-two percent of the patients achieved excellent results (CI $>80), 33 \%$ good results (CI $75-80)$, and $5 \%$ (1 patient) poor results $(\mathrm{CI}<75)$.

To further assess global changes in head shape after surgery, composite 3D images were generated for the preoperative, prehelmet, and posthelmet time points. Figure 4 depicts overlays of the preoperative, prehelmet, and endof-treatment composite images to demonstrate the change in head shape occurring from the preoperative to the 


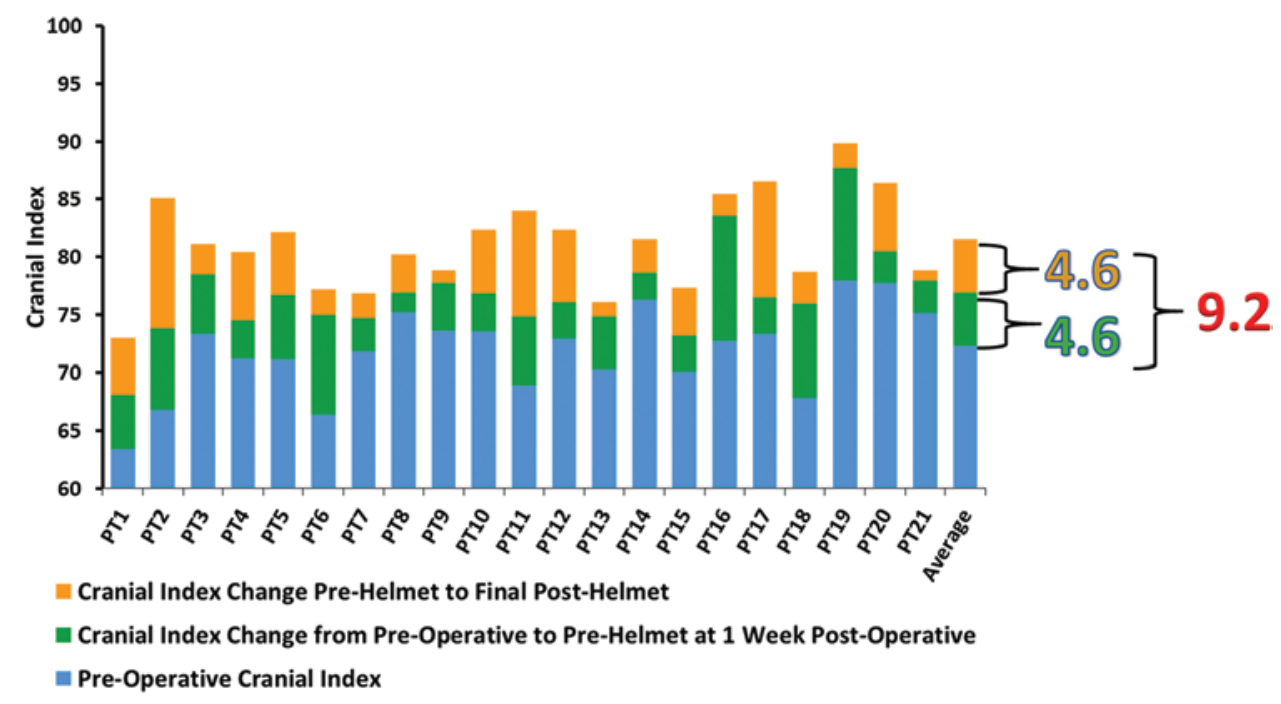

FIG. 1. Cranial index change for each subject. The starting $\mathrm{Cl}$ is shown in blue, the change in the $\mathrm{Cl}$ between surgery and the start of postoperative helmet therapy appears in green, and the change in the $\mathrm{Cl}$ while wearing the helmet appears in orange. The mean changes are displayed on the far right of the figure. PT = patient. Figure is available in color online only.

1-week postoperative (prehelmet) to the end-of-treatment time points. Color maps were generated to visually quantify the distance between the surfaces of the preoperative and end-of-treatment composite 3D images (Fig. 5).

The average maximum distance between the surfaces of the preoperative and 1-week postoperative 3D images was $13.0 \pm 4.1 \mathrm{~mm}$ (range $8.1-25.2 \mathrm{~mm}$ ). The average maximum distance between the surfaces of the preoperative and end-of-treatment 3D images was $24.7 \pm 6.8 \mathrm{~mm}$ (range 3.5-33.7 mm). Figure 6 shows a dot plot of the location of the maximum change in head shape for each subject between the preoperative 3D image and the 1-week postoperative 3D image (blue) and between the preoperative 3D image and the end-of-treatment 3D image (red).

\section{Discussion}

The purpose of this study was to analyze the change in head shape occurring after extended sagittal strip craniectomy with wedge ostectomies and postoperative helmet therapy using CI and 3D analysis. Cephalic index was used as a benchmark because of its long-standing use in previous studies for grading outcomes of treatment for sagittal synostosis. Three-dimensional analysis was employed to assess global changes in head shape occurring after surgery.

We found that CI changes rapidly after the surgical procedure, with $50 \%$ of the mean total change in CI (4.6) occurring by 7 days after surgery, before helmet therapy is initiated (Fig. 1). The mean time to stabilization of the CI was 57.2 days after surgery (Fig. 2). We found that $95 \%$ of the patients achieved a $C I \geq 75$. Figure 3 features representative photographs of a patient before and after surgery with a change in CI equal to the mean CI change of 9.2.

Variability in the ratio of change in the CI was observed between the immediate postoperative period (before helmet therapy) and during helmet therapy (Fig. 2 upper). The possible reasons for this difference may in- clude minor variations in the surgical technique between surgeons, variability in preoperative head shape, and/or variability in helmet design and management. Our study
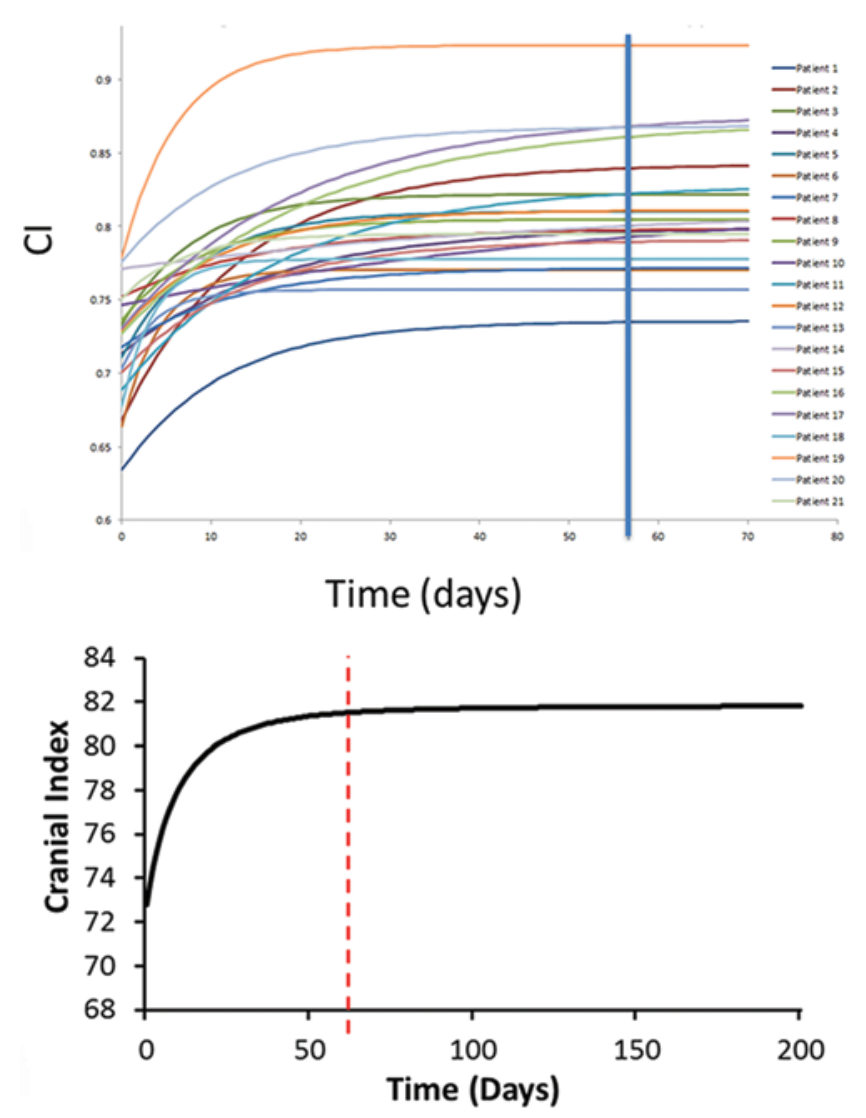

FIG. 2. Upper: The change in the $\mathrm{Cl}$ over time for each subject. Lower: The mean change in the $\mathrm{Cl}$ over time, with stabilization of the $\mathrm{Cl}$ at 57 days after surgery. Figure is available in color online only. 

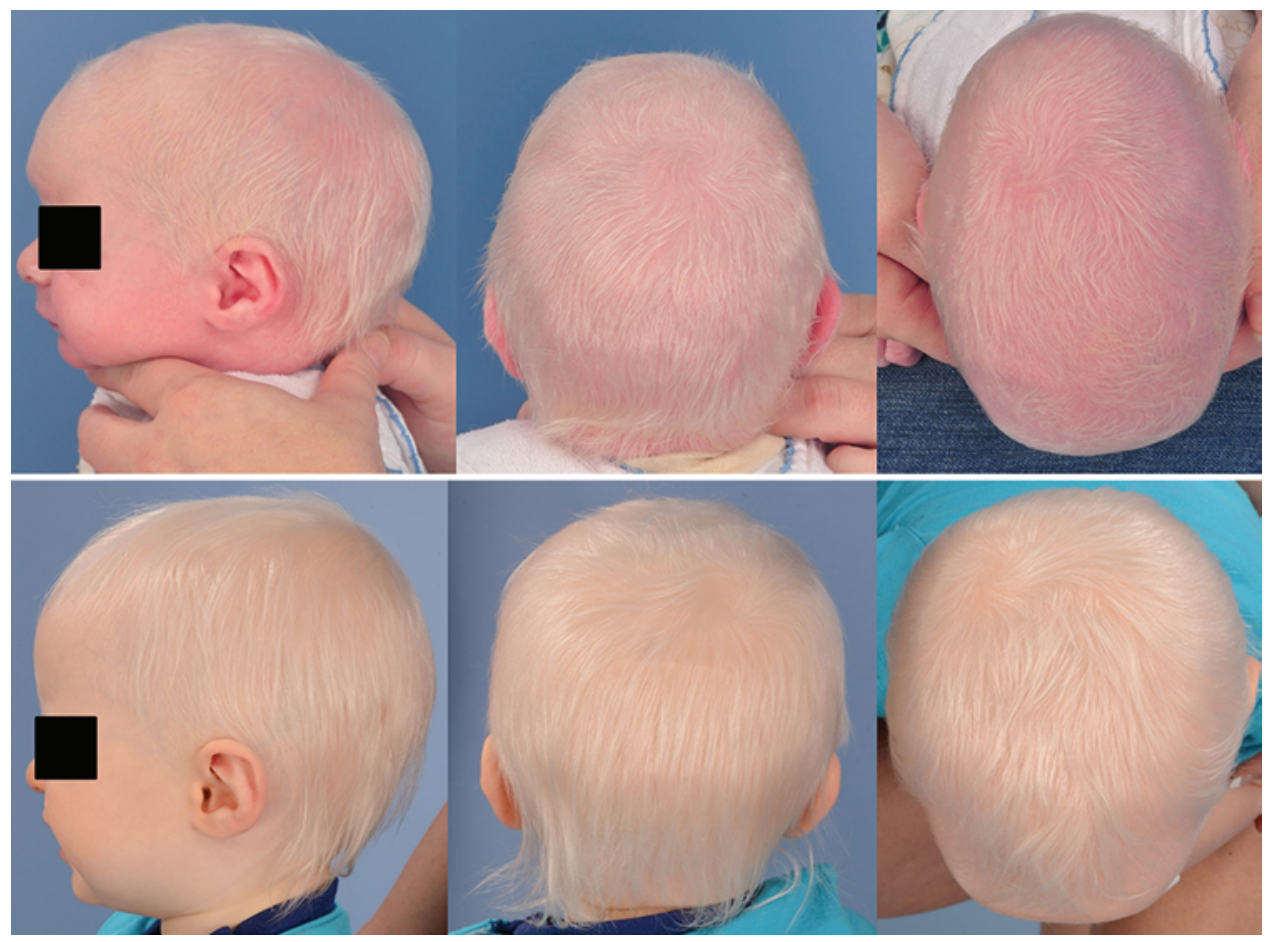

FIG. 3. Preoperative (upper) and end-of-helmet-therapy (lower) images obtained in a patient treated with extended sagittal strip craniectomy with wedge ostectomies. The patient's preoperative $\mathrm{Cl}$ was 0.71 and postoperative $\mathrm{Cl}$ was 80 . Figure is available in color online only.

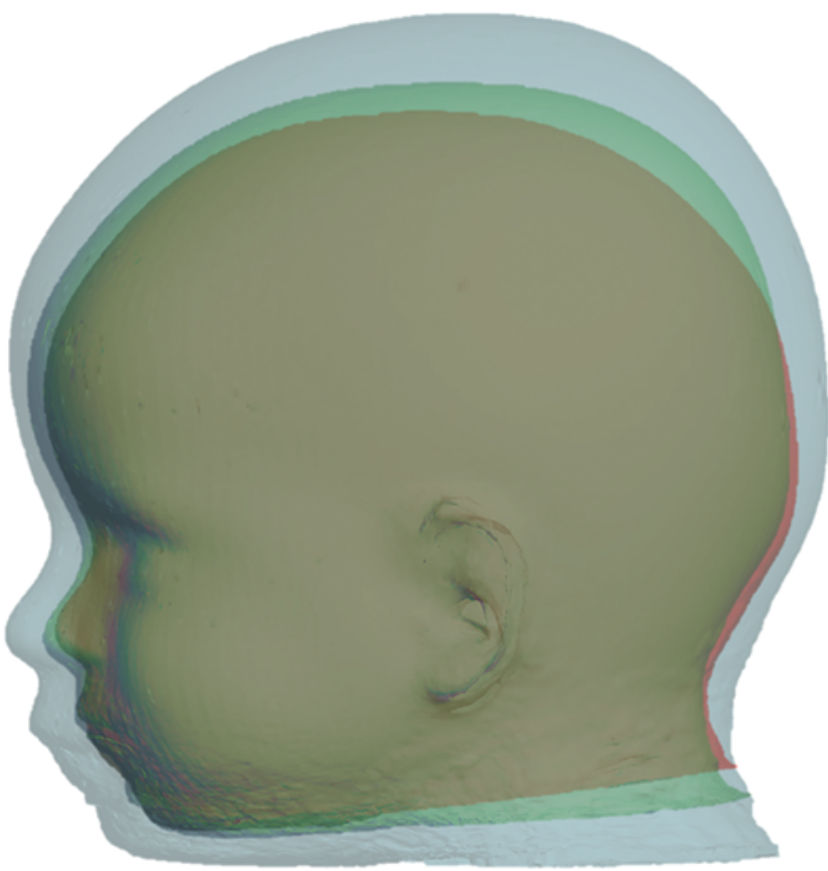

FIG. 4. Composite images were created for the preoperative (red), 1-week postoperative (green), and end-of-treatment (blue) time points. Note the significant increase in the vertical dimension of the posterior vault in both the immediate and long-term postoperative periods, with normalization of the vertex position. Figure is available in color online only. is not sufficiently powered to determine the significance of these individual variables with regard to their impact on the speed of change in CI. Statistical analysis did not show any correlation between the severity of preoperative $\mathrm{CI}$ and the rate or magnitude of change in CI.

The mean CI stabilized at 57.2 days after surgery (Fig. 2 lower), results consistent with those in a previous report by Jimenez et al. ${ }^{6}$ This time point may reflect a homeostasis being achieved between the opposing forces of the scalp and remodeling bone and the growing brain. This rapid change and stabilization in CI suggests that a shorter period of helmet therapy should be considered.

The duration of helmet therapy varies significantly among institutions. Jimenez et al. prescribe helmet therapy until 18 months of age and the use of 3 helmets during this period. ${ }^{6}$ Proctor prescribes the helmet for an average of 7-8 months and typically uses a single helmet. ${ }^{13}$ We currently prescribe helmet therapy until an age of 12-15 months old, utilizing 2-3 helmets, believing that sufficient growth is complete by this age to retain the corrected head shape. A randomized prospective study is warranted to explore whether shortening the duration of helmet therapy negatively affects head shape outcomes. Incrementally decreasing the duration of helmet therapy in serial cohorts would allow a threshold of change in head shape outcomes to be detected while minimizing the number of patients with compromised outcomes from surgery.

Cephalic index is a useful barometer for grading the severity of scaphocephaly in sagittal synostosis; however, it provides little objective data about head shape and whether that shape is perceived as normal. Dvoracek et al. 


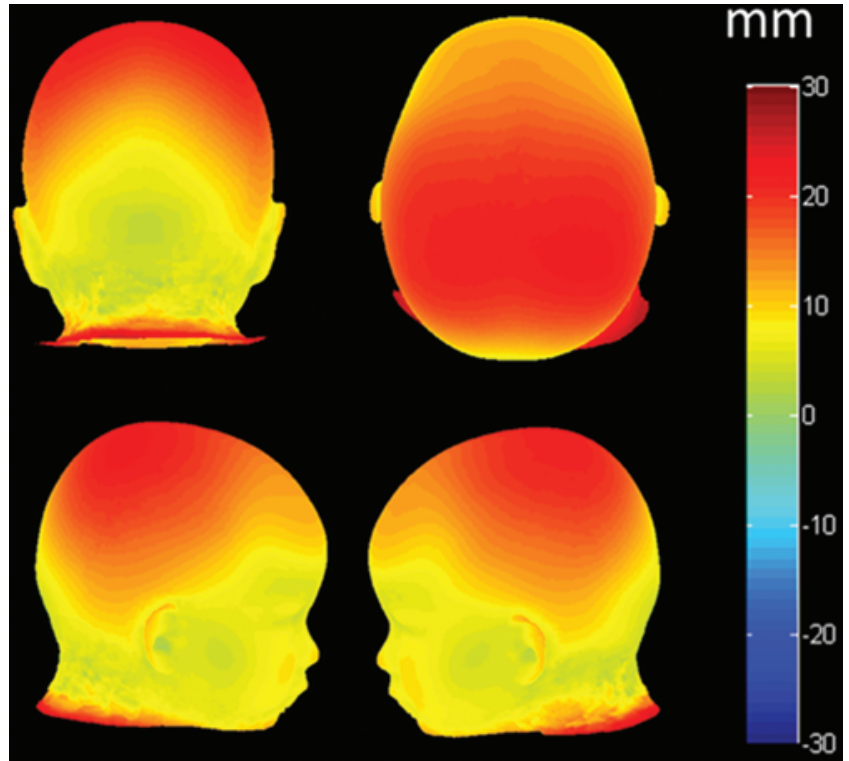

FIG. 5. Color maps were created to demonstrate the distance between the surfaces of the preoperative and end-of-treatment composite images. Scale shows the color correlation to distance. As shown in red, the greatest distances between the images occur in the vertical and transverse directions in the parietal regions.

recently reported that improved CIs were achieved after sagittal strip craniectomy, but that the maximal transverse dimension of the skull (euryon) remained in an abnormal location after surgery. ${ }^{2}$ Thus, CI can normalize while the shape of the cranial vault remains abnormal. We sought to further define changes in head shape in our patients after sagittal strip craniectomy using 3D imaging techniques.

Composite images were generated from the 3D images of the 21 subjects at the preoperative, prehelmet (1-week postoperative), and end-of-treatment time points. Figure 4 shows the overlay of the preoperative, prehelmet, and endof-treatment composite images, demonstrating the significant change in head shape that occurs during treatment. Over time, the vertex assumes a more normal posterior position due to significant vertical expansion and remodeling of the posterior cranial vault.

The color maps in Fig. 5 show the areas of greatest change between the preoperative and end-of-treatment images in red. There is a relatively even distribution in the magnitude of maximal change in the posterior cranial vault in the transverse and vertical dimensions. The dot plot in Fig. 6 demonstrates an even distribution of maximum change in the vertical and transverse vectors in the posterior cranial vault for both the 1-week postoperative and end-of-treatment time points.

The purpose of the wedge ostectomies is to facilitate lateral movement of the parietal bone flaps. After the bone cuts are made, there is an immediate transverse expansion of the posterior cranial vault that is appreciable before the incisions are closed; thus, the increased transverse dimension of the cranial vault in the immediate postoperative period was an expected finding. However, the increased vertical dimension of the posterior vault at the immediate postoperative (prehelmet) time point was unexpected.

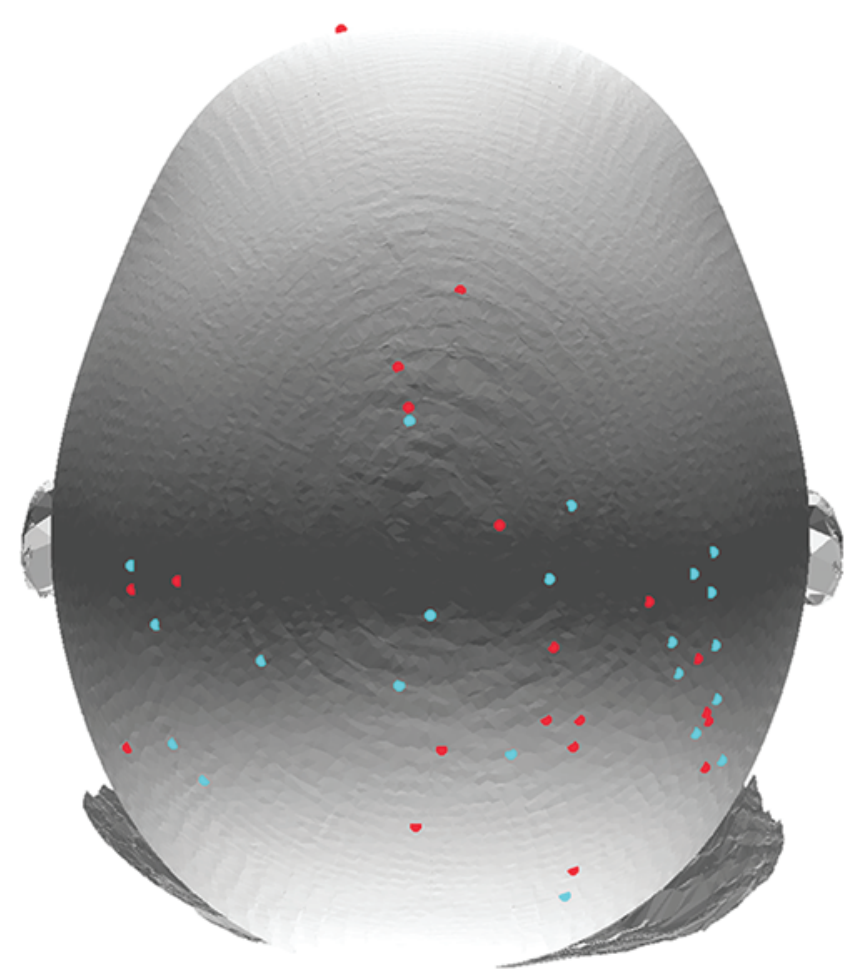

FIG. 6. A dot plot demonstrating the maximum change for each patient and the location of that change. Blue dots represent the maximum change between the preoperative and 1-week postoperative 3D images. Red dots indicate the location of maximum change between the preoperative and end-of-treatment images. Figure is available in color online only.

In the initial postoperative period, the patients continue to lie with their head positioned to one side because of the persistently narrow occiput of the scaphocephalic head. The vertical expansion observed before the helmet is applied is presumably attributable to the passive expansion of the brain and dura mater through the aperture created by the wide strip craniectomy.

Once applied, the helmet facilitates bilateral transverse expansion by transferring the weight of the head from the parietal bone flaps to the occiput. Figures 4-6 demonstrate the occipital remodeling and transverse and vertical expansion that occur during postoperative helmet therapy. The continued transverse hinging of the parietal bone flaps further increases the transverse dimension of the craniectomy defect, thus increasing the size of the aperture through which the dura can vertically expand. The vertical expansion itself can push the mobile parietal bone flaps laterally; thus, the vertical and transverse expansion and growth of the brain may synergistically drive the remodeling observed in this study.

A side-by-side comparison of subjects treated with and without wedge ostectomies was not performed; therefore, the direct impact of the wedge ostectomies alone cannot be determined in this study. It would be interesting to compare the vertical changes that accompany procedures that utilize narrower strip craniectomies with or without wedge ostectomies to see if comparable changes occur in the vertical dimension. Long-term 3D studies are needed 
to assess the durability of the head shape changes with continued growth.

One limitation of the current study is its sample size. Another lies in our inclusion criterion of patients treated by a single orthotist. We included only patients treated by the orthotist who manages the majority of our patients to control for any variability between multiple orthotists and their approach to and experience with postoperative helmet therapy.

\section{Conclusions}

After extended sagittal strip craniectomy with wedge ostectomies and postoperative helmet therapy, the CI normalizes rapidly (57.2 days) with an equal distribution of change in the CI occurring in the 1st week after surgery (prehelmet) and during helmet therapy. This finding may indicate a role for shortening the duration of helmet therapy. Three-dimensional analysis demonstrated significant expansion of the posterior cranial vault in the vertical and transverse dimensions. Further 3D studies are needed to determine how the width of the strip craniectomy and the presence or absence of wedge ostectomies affect 3D head shape outcomes.

\section{References}

1. David LR, Proffer P, Hurst WJ, Glazier S, Argenta LC: Spring-mediated cranial reshaping for craniosynostosis. J Craniofac Surg 15:810-818, 2004

2. Dvoracek LA, Skolnick GB, Nguyen DC, Naidoo SD, Smyth MD, Woo AS, et al: Comparison of traditional versus normative cephalic index in patients with sagittal synostosis: measure of scaphocephaly and postoperative outcome. Plast Reconstr Surg 136:541-548, 2015

3. Gociman B, Marengo J, Ying J, Kestle JR, Siddiqi F: Minimally invasive strip craniectomy for sagittal synostosis. J Craniofac Surg 23:825-828, 2012

4. Guimarães-Ferreira J, Gewalli F, David L, Olsson R, Friede H, Lauritzen CG: Spring-mediated cranioplasty compared with the modified pi-plasty for sagittal synostosis. Scand J Plast Reconstr Surg Hand Surg 37:208-215, 2003

5. Jimenez DF, Barone CM: Endoscopy-assisted wide-vertex craniectomy, "barrel-stave" osteotomies, and postoperative helmet molding therapy in the early management of sagittal suture craniosynostosis. Neurosurg Focus 9(3): e2, 2000

6. Jimenez DF, Barone CM, McGee ME, Cartwright CC, Baker CL: Endoscopy-assisted wide-vertex craniectomy, barrel stave osteotomies, and postoperative helmet molding therapy in the management of sagittal suture craniosynostosis. $\mathbf{J}$ Neurosurg 100 (5 Suppl Pediatrics):407-417, 2004

7. Khechoyan D, Schook C, Birgfeld CB, Khosla RK, Saltzman $\mathrm{B}$, Teng CC, et al: Changes in frontal morphology after single-stage open posterior-middle vault expansion for sagittal craniosynostosis. Plast Reconstr Surg 129:504-516, 2012

8. Lane L: Pioneer craniectomy for relief of mental imbecility due to premature sutural closure and microcephalus. J Am Med Assoc 18:49-50, 1892
9. Lannelongue M: De la craniectomie dans la microcephalie. Compt Rend Seances Acad Sci 50:1382-1385, 1890

10. Le MB, Patel K, Skolnick G, Naidoo S, Smyth M, Kane A, et al: Assessing long-term outcomes of open and endoscopic sagittal synostosis reconstruction using three-dimensional photography. J Craniofac Surg 25:573-576, 2014

11. Marcus JR, Domeshek LF, Loyd AM, Schoenleber JM, Das RR, Nightingale RW, et al: Use of a three-dimensional, normative database of pediatric craniofacial morphology for modern anthropometric analysis. Plast Reconstr Surg 124:2076-2084, 2009

12. Proctor MR: Endoscopic craniosynostosis repair. Transl Pediatr 3:247-258, 2014

13. Ridgway EB, Berry-Candelario J, Grondin RT, Rogers GF, Proctor MR: The management of sagittal synostosis using endoscopic suturectomy and postoperative helmet therapy. J Neurosurg Pediatr 7:620-626, 2011

14. Shah MN, Kane AA, Petersen JD, Woo AS, Naidoo SD, Smyth MD: Endoscopically assisted versus open repair of sagittal craniosynostosis: the St. Louis Children's Hospital experience. J Neurosurg Pediatr 8:165-170, 2011

15. Taylor JA, Maugans TA: Comparison of spring-mediated cranioplasty to minimally invasive strip craniectomy and barrel staving for early treatment of sagittal craniosynostosis. J Craniofac Surg 22:1225-1229, 2011

16. Toma R, Greensmith AL, Meara JG, Da Costa AC, Ellis LA, Willams SK, et al: Quantitative morphometric outcomes following the Melbourne method of total vault remodeling for scaphocephaly. J Craniofac Surg 21:637-643, 2010

17. van Veelen MLC, Mathijssen IM: Spring-assisted correction of sagittal suture synostosis. Childs Nerv Syst 28:1347-1351, 2012

\section{Disclosures}

The authors report no conflict of interest concerning the materials or methods used in this study or the findings specified in this paper.

\section{Author Contributions}

Conception and design: Derderian, Chou, Hallac, Kane. Acquisition of data: Chou, Hallac, Patel, Stewart, Smartt, Seaward. Analysis and interpretation of data: Derderian, Chou, Hallac, Patel, Cho. Drafting the article: Derderian, Chou. Critically revising the article: Derderian, Chou. Reviewed submitted version of manuscript: all authors. Approved the final version of the manuscript on behalf of all authors: Derderian. Study supervision: Derderian, Hallac.

\section{Supplemental Information}

\section{Previous Presentations}

This work was presented at the 2016 American Society of Plastic Surgeons Annual Meeting, Los Angeles, California.

\section{Correspondence}

Christopher Derderian, Department of Plastic Surgery, University of Texas Southwestern Medical Center, 1801 Inwood Rd., Dallas, TX 75390-9132. email: christopher.derderian@utsouthwestern. edu. 